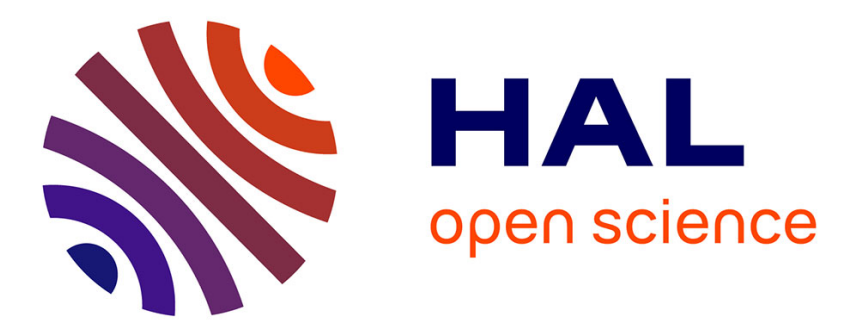

\title{
Interoception and embodiment in patients with bilateral vestibulopathy
}

Estelle Nakul, Charles Dabard, Michel Toupet, Charlotte Hautefort, Christian van Nechel, Bigna Lenggenhager, Christophe Lopez

\section{- To cite this version:}

Estelle Nakul, Charles Dabard, Michel Toupet, Charlotte Hautefort, Christian van Nechel, et al.. Interoception and embodiment in patients with bilateral vestibulopathy. Journal of Neurology, 2020, 10.1007/s00415-020-10221-x . hal-02988481

\section{HAL Id: hal-02988481 \\ https://hal-amu.archives-ouvertes.fr/hal-02988481}

Submitted on 4 Nov 2020

HAL is a multi-disciplinary open access archive for the deposit and dissemination of scientific research documents, whether they are published or not. The documents may come from teaching and research institutions in France or abroad, or from public or private research centers.
L'archive ouverte pluridisciplinaire HAL, est destinée au dépôt et à la diffusion de documents scientifiques de niveau recherche, publiés ou non, émanant des établissements d'enseignement et de recherche français ou étrangers, des laboratoires publics ou privés. 


\section{Interoception and embodiment in patients with 2 bilateral vestibulopathy}

3

4

5 Estelle $\mathrm{Nakul}^{1}$, Charles Dabard ${ }^{1}$, Michel Toupet ${ }^{2,3}$, Charlotte Hautefort ${ }^{2,4}$, Christian van 6 Nechel $^{2,5,6,7}$, Bigna Lenggenhager ${ }^{8 \#} \&$ Christophe Lopez ${ }^{1{ }^{1 *}}$ 7

$8{ }^{1}$ Aix Marseille Univ, CNRS, LNSC, FR3C, Marseille, France

$9{ }^{2}$ IRON, Institut de Recherche en Oto-Neurologie, Paris, France

$10{ }^{3}$ Centre d'Explorations Fonctionnelles Oto-Neurologiques, Paris, France

$11{ }^{4}$ Service ORL, Hôpital Lariboisière, Paris, France

$12 \quad{ }^{5}$ Unité Troubles de l'Équilibre et Vertiges, CHU Brugmann, Bruxelles, Belgium

$13{ }^{6}$ Unité de Neuro-Ophtalmologie, CHU Erasme, Bruxelles, Belgium

$14{ }^{7}$ Clinique des Vertiges, Bruxelles, Belgium

15 16 17 18 19

${ }^{8}$ Cognitive Neuropsychology, Department of Psychology, University of Zurich, Switzerland

*ORCID ID (E. Nakul): https://orcid.org/0000-0001-8707-3998

*ORCID ID (B. Lenggenhager): https://orcid.org/0000-0003-0418-9931

*ORCID ID (C. Lopez): https://orcid.org/0000-0001-9298-2969

\#Shared last authorship. These authors contributed equally to the present study.

Number of figures: 4; Number of tables: 1

*Corresponding author:

Dr Christophe Lopez

Laboratoire de Neurosciences Sensorielles and Cognitives - UMR 7260

Aix Marseille Univ \& Centre National de la Recherche Scientifique (CNRS)

Centre Saint-Charles, Fédération de Recherche 3C - Case B

3, Place Victor Hugo. 13331 Marseille Cedex 03, France

Tel: +33413550841 Fax: + 33413550844 Email: christophe.lopez@univ-amu.fr 


\section{Abstract}

34 There are tight functional and anatomical links between the vestibular and interoceptive systems, and both 35 systems have shown to fundamentally underlie emotional processes and our sense of a bodily self. Yet, 36 nothing is known about how long-term bilateral vestibulopathy (BVP) influences interoception and its 37 relation to embodiment and the sense of self. We thus compared cardiac interoceptive accuracy, confidence 38 in the performance, and general body awareness in 25 BVP patients and healthy controls using a heartbeat 39 tracking task, self-reports about interoceptive awareness, as well as measures of self-localization and of 40 self-body closeness. Results showed no difference between patients and controls regarding interoceptive 


\section{Introduction}

70 Vestibular disorders are typically associated with deficits in balance and eye movements control. In 71 addition to these deficits in reflexes, higher-level functions are often affected [1]. While most research has 72 focused on spatial cognition, recent evidence in healthy participants suggests a vestibular contribution to 73 cognitive and emotional functions, such as own-body perception, mood, empathy, and decision making 74 [2]. In line with this, clinical studies showed that patients with mainly unilateral peripheral vestibular 75 disorders report increased separation of the self from the body, symptoms of depersonalization and 76 derealization (reviewed in [3]), as well as higher anxiety and depression (reviewed in [4]). Yet, the mechanisms underlying such deficits remain poorly understood, and it has been emphasized that further investigations are needed to better understand the full spectrum of high-level disorders associated with peripheral vestibular deficits, in particular with bilateral vestibulopathy (BVP) [5].

One way through which vestibular disorders may affect own-body and self-perception as well as emotional processes might be a change in the ability to perceive the internal states of the body. Interoception is the sense of the internal physiological condition [6,7], originating from receptors in the gastrointestinal tract, kidneys, heart, blood vessels, etc. Interoception underpins emotional processes [7], and like the vestibular system, it is fundamental to the multisensory mechanisms underlying own-body and self perception [8-10]. Importantly, the vestibular and interoceptive systems are strongly interconnected at both anatomical and functional levels. Studies in animals showed that vestibular signals project to areas processing interoceptive signals in the brainstem, such as in the parabrachial nuclei, and project to the insular cortex [11]. Conversely, interoceptive signals - such as those generated by mechanical stimulation of visceral receptors - can modulate the spontaneous firing rate of vestibular nuclei neurons [12]. At a functional level, both vestibular and interoceptive systems inform about self-motion and gravity [11] and they interact to adapt blood pressure [13] and respiratory pattern [14] to own body movement and orientation. However, direct studies investigating the link between vestibular and interoceptive processes are to our knowledge lacking in the field of self-perception. It remains especially unclear to what degree BVP influences interoception and its relation to embodiment and the sense of self.

The present study primarily aims at evaluating the influence of long-term BVP on interoception. We measured the ability to perceive interoceptive signals using the heartbeat tracking task (HBTT), during which participants estimated the number of heartbeats felt during a given time interval $[15,16]$. The hypothesis about how BVP influences cardiac interoception is two-fold in that there is evidence to suggest either an increase in interoceptive sensitivity or a decrease in interoceptive sensitivity. On the one hand, considering that both vestibular and interoceptive signals contribute to own-body perception, BVP may result in a higher reliance on interoceptive signals to maintain stable the perceived anchoring of the self to the body, thus resulting in enhanced interoceptive sensitivity. Cross-modal plasticity and higher awareness for intact sensory systems have been observed in many instances of sensory deprivation (reviewed in [17]), such as in individuals with congenital blindness, showing higher odor awareness and lower odor detection 
threshold [18]. This mechanism might be prominent in the perception of gravity, shown to be crucial for

the experienced self-location: BVP patients missing head-centered vestibular organs may rely more on information from trunk-centered inner organs typically linked to interoception [19]. On the other hand, the loss of vestibular signals may alter the processing of interoceptive signals in multisensory brainstem and cortical regions, thus decreasing interoceptive sensitivity. In line with this hypothesis is evidence of poorer cardiac interoception in patients with olfactory deficits [20] and in patients with spinal cord injury [21] when compared to healthy participants.

The secondary aim of the study is to evaluate the relation between the vestibular system, cardiac interoception and the sense of embodiment. We measured interoceptive awareness using the Body Perception Questionnaire (BPQ) [22]. BPQ scores have been positively correlated with gray matter volume in the right anterior insula, a core region for interoception [23]. Furthermore, we tested two aspects of selflocation and analyzed them in relation to interoceptive abilities: (1) Where in the body is the self perceived, and (2) How strong is the anchoring of the self to the body. Self-location can be measured by asking participants to point on a human silhouette [24, 25]. Studies showed that most participants locate their self either in their head or trunk. Head-locators and trunk-locators may differ on their interoceptive abilities and personality traits $[26,27]$. How strong the self is perceived to be anchored to the body can be measured by pictorial descriptions of self-body closeness [28]. We hypothesized that patients with BVP may report self-location differently than controls, potentially more around the trunk, a major egocentric reference frame whose importance might be further increased through higher reliance on somatosensory [29] and interoceptive signals.

\section{Methods}

\section{Participants}

128 We tested 25 patients with a BVP and 25 healthy controls matched for sex, age, education and body-mass index (Table 1). All patients had weak responses to a bithermal caloric test with water at $44^{\circ} \mathrm{C}$ and $30^{\circ} \mathrm{C}$ (mean slow phase eye velocity $<5 \%$ s; left ear (mean \pm SD): $44^{\circ} \mathrm{C}: 1.4 \pm 2.3 \%$ s, $30^{\circ} \mathrm{C}: 2.0 \pm 2.6^{\circ} / \mathrm{s}$; right ear: $44^{\circ} \mathrm{C}: 1.8 \pm 2.7^{\circ} / \mathrm{s}, 30^{\circ} \mathrm{C}: 2.5 \pm 5.3^{\circ} / \mathrm{s}$ ) and reduced responses to the video head impulse test (gain < 0.7 ; horizontal canals: $0.33 \pm 0.27$; anterior canals: $0.26 \pm 0.23$; posterior canals: $0.27 \pm 0.24$ ). Otolithic functions were evaluated by cervical vestibular-evoked myogenic potentials (cVEMPs) over the sternocleidomastoid muscles and ocular vestibular-evoked myogenic potentials (oVEMPs) over the inferior oblique muscles. cVEMPs were present in the left ear for 9 patients (p13-n23 amplitude: $104 \pm 36$ $\mu \mathrm{V})$ and in the right ear for 13 patients $(75 \pm 47 \mu \mathrm{V})$. oVEMPs were present in the left ear for 5 patients $(2.3 \pm 1.4 \mu \mathrm{V})$ and in the right ear for 6 patients $(3.4 \pm 1.0 \mu \mathrm{V})$. Controls had no history of vestibular, neurological, or psychiatric disease. All patients were right-handed (Edinburgh Handedness inventory: 97 $\pm 6 \%), 23$ controls were right-handed $(89 \pm 13 \%)$ and 2 were left-handed (-100\%). All participants had normal or corrected-to-normal vision. Anxiety and depression measured with the Hospital Anxiety and 
141 Depression scale [30] did not differ between patients and controls (Table 1). All participants were informed about the study and gave written informed consent. Procedures were approved by the Ethics Committee and followed the Declaration of Helsinki.

\section{Heartbeat tracking task: procedures and analyses}

Participants performed the HBTT following established procedures [16]. They sat comfortably on a stool with their arms dangling and counted the heartbeats they felt between two identical sounds, defining six time intervals of $25,30,35,40,45$ and $50 \mathrm{~s}$. They were instructed to count silently, without checking their heartbeat manually by pressing a body part on the floor or against their seat. The experimenter controlled that they followed the instructions. After each second sound, participants verbally indicated how many heartbeats they perceived. The six time intervals were presented once in a randomized order using Superlab 4.5 (Cedrus, San Pedro, USA). Participants could take a break for as long as they wanted between trials. Heartbeats were simultaneously recorded using FLAT Active electrodes (Biosemi, Amsterdam, The Netherlands) placed on their sternum and left chest. For each time interval, an interoceptive accuracy score was calculated using the following formula [16]:

$$
1-\frac{\mid \text { Recorded Heart Beats - Counted Heart Beats } \mid}{\text { (Counted Heart Beats + Recorded Heart Beats) } / 2}
$$

Interoceptive accuracy scores can range from -1 to 1 , indicating very poor to perfect performance. A mean interoceptive accuracy score was calculated for each participant by averaging the accuracy scores over the six time intervals.

Immediately after each trial, participants rated the confidence in their judgment on a vertical, continuous visual analogue scale, using pencil and paper. The upper part of the scale referred to "Completely confident (perfect perception of my heartbeats)" and the bottom part to "Random answer (no awareness of my heartbeats)". For each time interval, confidence ratings were measured in $\mathrm{mm}$ and converted in percentage (Random answer: 0\%; Completely confident: 100\%). A mean confidence score was calculated for each participant by averaging the accuracy scores over the six time intervals.

\section{Body awareness}

Participants rated 45 items from the body awareness subscale of the BPQ [22]. They rated how aware they are of their bodily processes using a five-point scale ranging from "never" to "always". One patient did not answer the questionnaire.

\section{Self-location}

Self-location was measured by a pointing task [31] on a computer screen $(1366 \times 768$ pixels $)$. After they read the instructions "Where do you locate your 'self"? On this picture representing a body, indicate the location (a single point) which according to you best represents the location of the self", participants were shown a human silhouette of their own sex, once from the front, once from the back, and once from the profile (Figure 2A), in a randomized order. With a computer mouse in their dominant hand they clicked on a point of the silhouette that best described the location of their self. Instructions and pictures were 
presented with PsychoPy [32], which also recorded the coordinates of pointing. One control did not complete the task.

\section{Self-body closeness}

Participants reported the perceived closeness between self-body, self-immediate body environment, body-immediate body environment, and self-others using simple pictorial descriptions [28] adapted from the Inclusion of Other in the Self scale [33] (Figure 3). They reported the average perception over the last year. Participants selected 1 out of 7 pictorial descriptions of the closeness between, e.g., their self and body, whereby two distinct circles represent clear separation between their self and body (score $=1$ ) and two overlapping circles represent maximal closeness between their self and body (score $=7$ ).

\section{Organization of a session}

Participants completed the HBTT, BPQ, self-location and self-body closeness tasks in a random order.

\section{Statistical analyses}

As most of the dependent variables were not normally distributed (Shapiro-Wilk test), we used nonparametric procedures (SPSS 25, IBM, USA): inter-group differences were analyzed using Mann-Whitney $\mathrm{U}$ tests and Spearman correlations were used to analyze links between self-body closeness and cardiac interoception. Differences were considered significant for $\mathrm{p}<0.05$.

\section{Results}

\section{Interoceptive accuracy, confidence and body awareness}

We found no significant difference between patients and controls regarding their interoceptive accuracy $(U=261.5, Z=-0.99, p=0.32)$ and confidence in their judgment $(U=287.0, Z=-0.50, p=0.62$; Fig. 1A). Similarly, body awareness as measured by BPQ scores did not differ significantly between patients and controls $(U=287.0, Z=-0.02, p=0.98$; Fig. 1B $)$.

\section{Self-location and its relation to cardiac interoception}

Results from the pointing task revealed no significant difference in the coordinates of self-location between patients and controls for the front $(x: U=246.0, Z=-1.49, p=0.14 ; y: U=273.0, Z=-0.98, p=0.33$ ), back (x: $U=290.0, Z=-0.66, p=0.51 ; y: U=308.0, Z=-0.32, p=0.75)$ and side $(x: U=257.0, Z=-$ $1.28, p=0.20 ; y: U=296.0, Z=-0.55, p=0.59)$ views of the body (Fig. 2A).

In a second step, patients and controls were allocated to the head-locator and trunk-locator groups, corresponding to any self-location above or below the neck, respectively: 12 patients and 13 controls were head-locators; 13 patients and 11 controls were trunk-locators (Fig. 2B). The proportion of head- and trunk-locators was similar in both groups $(\chi=0.19, \mathrm{p}=0.78)$. Head- and trunk-locators did not differ regarding their interoceptive accuracy (patients: $U=69.5, Z=-0.46, p=0.64$; controls: $U=67.0, Z=-$ $0.26, p=0.79$ ), confidence judgment (patients: $U=66.0, Z=-0.65, p=0.51$; controls: $U=47.5, Z=1.39$, 
$\mathrm{p}=0.16$ ) and body awareness (patients: $\mathrm{U}=60.5, \mathrm{Z}=-0.64, \mathrm{p}=0.52$; controls: $\mathrm{U}=51.5, \mathrm{Z}=-0.89, \mathrm{p}=$ 0.37) (Fig. 2C). In addition, there was no difference between patients and controls from the head-location group (interoceptive accuracy: $U=54.5, Z=-1.28, p=0.20$; confidence: $U=68.5, Z=-0.52, p=0.60$; body awareness: $U=76.0, Z=-0.05, p=0.96$ ) and no difference between patients and controls from the trunk-location group (interoceptive accuracy: $U=69.0, Z=-0.14, p=0.88$; confidence: $U=53.0, Z=-$ $1.07, p=0.28$; body awareness: $U=72.0, Z=-0.27, p=0.78$ ).

\section{Perceived self-body closeness and its relation to cardiac interoception}

Fig. 3 shows that patients and controls did not differ significantly regarding the perceived strength of closeness between their self and body $(U=250.0, Z=-0.83, p=0.41)$, self and immediate body environment $(U=285.5, Z=-0.05, p=0.96)$, body and immediate body environment $(U=262.0, Z=-$ $0.54, p=0.59)$, or self and others $(U=282.0, Z=-0.13, p=0.90)$. However, we note large interindividual variability in these judgments, which were explored in correlation analyzes.

Our exploratory analyzes significantly related self-body closeness and cardiac interoception. In the overall population, interoceptive accuracy correlated positively with self-body closeness $(\rho=0.31, \mathrm{p}$ $=0.03$ ), indicating that stronger anchoring of the self to the body was associated with better cardiac interoception (Fig. 4A). Conversely, interoceptive accuracy negatively correlated with self-environment closeness $(\rho=-0.29, \mathrm{p}=0.04)$, showing that lower anchoring of the self to the body was linked to lower cardiac interoception (Fig. 4B). Yet, when considering each group separately, interoceptive accuracy did not correlate with self-body closeness (patients: $\rho=0.28, \mathrm{p}=0.18$; controls: $\rho=0.37, \mathrm{p}=0.07$ ) and selfenvironment closeness (patients: $\rho=-0.35, \mathrm{p}=0.08$; controls: $\rho=-0.23, \mathrm{p}=0.26$ ).

In the overall population, body awareness (BPQ scores) was also positively correlated with selfbody closeness $(\rho=0.31, \mathrm{p}=0.03$; Fig. $4 \mathbf{C})$, pointing to a link between interoceptive awareness and embodiment. BPQ scores did not correlate with self-body closeness in the patients $(\rho=0.28, \mathrm{p}=0.19)$ and in the controls $(\rho=0.38, \mathrm{p}=0.07)$ when considered separately. Finally, in the whole population, selfbody closeness did not correlate with anxiety $(\rho=-0.23, \mathrm{p}=0.11)$ and depression $(\rho=-0.20, \mathrm{p}=0.16)$. However, self-body closeness was negatively correlated with anxiety $(\rho=-0.41, \mathrm{p}=0.04)$ and depression $(\rho=-0.54, \mathrm{p}=0.005)$ in the patients, but not the in controls (anxiety: $\rho=-0.16, \mathrm{p}=0.44$; depression: $\rho=$ $0.02, \mathrm{p}=0.93)$.

\section{Discussion}

The tasks used here revealed no difference between BVP patients and a carefully matched control group regarding their interoceptive accuracy, body awareness, self-location, and self-body closeness. Such negative findings are difficult to interpret and it cannot be excluded that differences could be found with other measures of interoception (see [34] for a critical account of the HBTT), or with larger samples. Nevertheless, our results help to better delineate the range of perceptual and cognitive deficits associated with BVP. The general trend to publish only positive results might bias the understanding of the patients' 
symptomatology. Negative findings should thus be reported more systematically in clinical neuroscience to avoid such bias. Furthermore, explorative correlative analyses revealed a relation between interoception and self-body closeness, which might be important to consider in future studies.

\section{No evidence for a change in cardiac interoception in bilateral vestibulopathy} suggesting that a long-lasting BVP does not change cardiac sensitivity or interoceptive awareness [35]. The results do not support the hypothesis of increased interoceptive sensitivity, suggesting that BVP does not involve higher focus on interoception through higher reliance on signals from trunk-centered inner organs. Likewise, the results do not support the hypothesis of decreased cardiac interoceptive sensitivity in BVP that may arise from altered interoceptive information processing in multisensory brainstem and cortical regions. Our findings contrast with alterations of cardiac interoception also measured with the HBTT in patients with olfactory [20] and sensorimotor deficits [21].

The lack of significant difference found in our study might be explained by several factors. First, the absence of effect of BVP on cardiac interoception may reflect a lack of relevance of vestibular signals for cardiac interoception or the poor interactions between these systems. This seems in contrast with the wealth of data on the anatomical and functional interplay between the vestibular and interoceptive systems, including the cardiovascular system $[12-14,36]$. There is, for example, evidence that stimulation of the semi-circular canals can change the respiratory frequency in healthy participants, whereas this effect is not consistent in patients with BVP [14]. Similarly, caloric vestibular stimulation changed heart rate variability and blood pressure variability in healthy participant, an effect which was absent in a patient with BVP [36]. Second, the absence of effect of BVP on cardiac interoception may be related to the fact that BVP patients were tested years after the onset of the disease. Future studies should test whether a similar result is found in the acute phase of the BVP. Third, we only assessed cardiac interoception, while other aspects of interoception, e.g. related to graviceptors in the viscera, have not been measured. It is still an open debate to what extent a common interoceptive ability exists and how much from cardiac interoception can be generalized to other interoceptive systems [37]. Future studies should therefore investigate the link between vestibular information processing and other interoceptive signals, such as respiratory or gastric signals. Although graviceptors in the viscera contribute to encode own-body orientation in healthy participants [38], it remains to be established whether this information is used differently in BVP patients and if it also contributes significantly to an embodied sense of self.

\section{No difference in perceived self-location and self-body closeness in bilateral vestibulopathy}

Self-reports showed no difference between patients and controls regarding where in their body the self was perceived and how strong the self was perceived as anchored to the body. The vestibular system has been described as crucially involved in perceiving self-location within the bodily borders (reviewed in [8]). A higher occurrence of sensations of detachment of the self from the body has been reported in a 
sample of 60 patients with mainly unilateral peripheral vestibular disorders than in sex- and age-matched healthy participants [39]. A prospective study in 210 patients suffering from vertigo and dizziness showed that full-blown out-of-body experiences were also more frequent in patients with documented unilateral peripheral vestibular disorders than in sex- and age-matched healthy participants [40]. Second, there is evidence that galvanic vestibular stimulation in healthy participants can modulate the anchoring of the self important for an embodied self-location, self-reports used in our study showed no effect of BVP on selflocation and on the perceived self-body closeness.

In acute peripheral vestibular disorders (and during artificial vestibular stimulation in healthy participants), the central nervous system receives signals from the inner ear about self-motion and selforientation that are incongruent with visual and somatosensory signals, thereby creating sensory mismatch and perceptual incoherence. By contrast, as vestibular information is missing in BVP patients, it does not conflict with (or confirm) visual and somatosensory encoding of self-motion. We argue that the absence of sensory mismatch in BVP may account for normal self-location and self-body closeness in the present study. In line with this idea, patients with a long history of BVP show similar abilities than controls to take another person's viewpoint, thus to mentally detached oneself from an embodied self-location [28]. By contrast, this perspective-taking task is influenced by vestibular stimulation on a rotatory chair in healthy participants [42], and is impaired in patients with unilateral vestibular failure (benign paroxysmal positional vertigo and acute vestibular neuritis) [43].

The self-localization task and the measure of self-body closeness yielded congruent results with previous studies in younger participants. As in previous studies, both groups localized their self either in their head or trunk [24, 25], and presented a similar degree of self-body closeness using an adaptation of the Inclusion of Other in the Self scale [28]. We also found a similar proportion of head-and trunk-locators. These interindividual differences in the perceived self-location have been related to cultural factors and personality traits [26]. Pioneering studies [e.g. 44] related self-location in the head to the origin of the multimodal, but mainly visual, first-person perspective. Self-location in the trunk, especially near the heart, 318 was found in participants characterizing themselves as emotional, feminine and interpersonally warm [26]. 319 Finally, we found no relation between head/trunk-location and interoceptive accuracy or body awareness, 320 and could therefore not support the hypothesis that trunk-locators used cardiac interoceptive information 321 differently.

\section{Higher self-body closeness is associated with better interoceptive accuracy}

Independent of the group, we found in an exploratory analysis, that interoceptive accuracy was positively correlated to self-body closeness and negatively correlated with self-environment closeness. This suggests that stronger anchoring of the self to the body, i.e. embodiment, is linked to better capacity to perceive and report cardiac interoceptive signals. In line with this finding, there is evidence that cardiac interoceptive accuracy can predict the malleability of body representations as measured by a multisensory illusion in 
healthy participants (i.e. the rubber hand illusion [45]). Furthermore, body awareness (BPQ scores) also increased with self-body closeness, supporting the idea that a stronger sense of embodiment co-occurs with higher awareness of signals from internal organs. Finally, we found that self-body closeness decreased with increasing anxiety and depression in BFV patients, but not in controls, despite the fact that controls and patients do not differ in their anxiety and depression score. A recent prospective study reported that anxiety was a predictor of out-of-body experiences in patients suffering from dizziness and vertigo, but not in healthy controls [40]. Altogether, these results highlight the interplay between anxiety, depression and depersonalization-derealization symptoms in patients with vestibular disorders $[40,46$, 47].

\section{Limitations of the study and perspectives}

Here, self-location was reduced to the coordinates of a single point in space, although self-location has also been defined as a volume occupied by the self in space [8], and has even been linked to peripersonal space [48]. Future studies should endeavor to measure self-location in BVP patients using tasks allowing 3D measures of self-location, e.g. using virtual reality [49], or measures of the peripersonal space boundaries. We also note that our negative findings may also be due to the fact that we used only selfreports to measure the perceived self-body closeness, rather than objective measures. Other measures have been proposed where participants explicitly evaluate their self-location and self-identification with an avatar shown in a head-mounted display in virtual reality. For example, when participants received a tactile stimulation on their back and observed an avatar being stroked at the same time on the same body region, they self-identified with the avatar and localized themselves closer to it (i.e., disembodied self-location) [49]. It will be important in future studies to investigate how BVP patients experience self-body closeness during experimentally-induced sensations of disembodied self-location.

\section{Conclusions}

The present study should help understand the complex symptomatology of patients with BVP, who present with deficits reaching far beyond the well-known imbalance and oscillopsia, including alterations of the most fundamental aspects of the self.

\section{Acknowledgements}

The authors declare no competing financial interests. The research leading to these results has received funding from the Swiss National Science Foundation (grant number: PP00P1_170511). 


\section{Figure Legends}

Figure 1. Cardiac interoception. (A) Interoceptive accuracy and confidence in the judgment for BVP patients ( $r e d$ ) and healthy controls (green). (B) Score to the body awareness scale of the Body Perception interquartile ranges (10-90). Means are shown as + and dots are outliers.

Figure 2. Self-location and its relation to cardiac interoception. (A) Self-location indicated by patients (red dots) and controls (green squares) on a human silhouette seen from the back, profile and front. (B) Histograms show the number of participants according to the y coordinates of self-location. (C) Interoceptive accuracy compared in head- and trunk-locators for the patients (red) and controls (green). Box and whiskers plots show medians and interquartile ranges (10-90). Means are shown as + and dots are outliers.

Figure 3. Reported self-body closeness. Seven pairs of circles at the bottom of the figure were presented to participants, who had to indicate which one better represented the perceived degree of closeness between two items, such as their self and body. Box and whiskers plots show medians and interquartile ranges (1090). Means are shown as + and dots are outliers.

Figure 4. Relation between self-body closeness, cardiac interoception and body awareness. Correlation between interoceptive accuracy and self-body closeness (A), between interoceptive accuracy and self-environment closeness (B) and between body awareness (BPQ score) and self-body closeness (C). 


\section{References}

1. Smith PF (2017) The vestibular system and cognition. Curr Opin Neurol 30:84-89. https://doi.org/10.1097/WCO.0000000000000403

2. Lopez C (2016) The vestibular system: balancing more than just the body. Curr Opin Neurol 29:74-83. https://doi.org/10.1097/WCO.0000000000000286

3. Jauregui-Renaud K (2015) Vestibular function and depersonalization/derealization symptoms. Multisens Res 28:637-651

4. Jacob RG, Furman JM (2001) Psychiatric consequences of vestibular dysfunction. Curr Opin Neurol 14:41-46

5. Lucieer F, Duijn S, Van Rompaey V, et al (2018) Full Spectrum of Reported Symptoms of Bilateral Vestibulopathy Needs Further Investigation-A Systematic Review. Front Neurol 9:. https://doi.org/10.3389/fneur.2018.00352

6. Craig AD (2003) Interoception: the sense of the physiological condition of the body. Curr Opin Neurobiol 13:500-505

7. Khalsa SS, Adolphs R, Cameron OG, et al (2018) Interoception and Mental Health: A Roadmap. Biol Psychiatry Cogn Neurosci Neuroimaging 3:501-513. https://doi.org/10.1016/j.bpsc.2017.12.004

8. Blanke O (2012) Multisensory brain mechanisms of bodily self-consciousness. Nat Rev Neurosci 13:556-571. https://doi.org/10.1038/nrn3292

9. Azzalini D, Rebollo I, Tallon-Baudry C (2019) Visceral Signals Shape Brain Dynamics and Cognition. Trends Cogn Sci (Regul Ed) 23:488-509. https://doi.org/10.1016/j.tics.2019.03.007

10. Park H-D, Blanke O (2019) Coupling Inner and Outer Body for Self-Consciousness. Trends Cogn Sci (Regul Ed) 23:377-388. https://doi.org/10.1016/j.tics.2019.02.002

11. Craig AD (2009) How do you feel-now? The anterior insula and human awareness. Nat Rev Neurosci 10:59-70. https://doi.org/nrn2555 [pii] 10.1038/nrn2555

12. Dutov AM (1974) Responses of neurons of the vestibular nuclei to interoceptive stimulation. Bulletin of Experimental Biology and Medicine 78:849-851

13. Balaban CD, Porter JD (1998) Neuroanatomic substrates for vestibulo-autonomic interactions. J Vestib Res 8:7-16

14. Jauregui-Renaud K, Gresty MA, Reynolds R, Bronstein AM (2001) Respiratory responses of normal and vestibular defective human subjects to rotation in the yaw and pitch planes. Neurosci Lett 298:17-20. https://doi.org/S0304394000017316 [pii]

15. Schandry R (1981) Heart beat perception and emotional experience. Psychophysiology 18:483488

16. Garfinkel SN, Seth AK, Barrett AB, et al (2015) Knowing your own heart: Distinguishing interoceptive accuracy from interoceptive awareness. Biological Psychology 104:65-74. 
https://doi.org/10.1016/j.biopsycho.2014.11.004

17. Frasnelli J, Collignon O, Voss P, Lepore F (2011) Crossmodal plasticity in sensory loss. Prog Brain Res 191:233-249. https://doi.org/10.1016/B978-0-444-53752-2.00002-3

18. Beaulieu-Lefebvre M, Schneider FC, Kupers R, Ptito M (2011) Odor perception and odor awareness in congenital blindness. Brain Research Bulletin 84:206-209. https://doi.org/10.1016/j.brainresbull.2010.12.014

19. Mittelstaedt H (1992) Somatic versus vestibular gravity reception in man. Ann N Y Acad Sci 656:124-39

20. Krajnik J, Kollndorfer K, Notter LA, et al (2015) The impact of olfactory dysfunction on interoceptive awareness. Psychophysiology 52:263-268. https://doi.org/10.1111/psyp.12316

21. Montoya P, Schandry R (1994) Emotional experience and heartbeat perception in patients with spinal cord injury and control subjects. J Psychophysiol 8:289-296

22. Cabrera A, Kolacz J, Pailhez G, et al (2018) Assessing body awareness and autonomic reactivity: Factor structure and psychometric properties of the Body Perception Questionnaire-Short Form (BPQ-SF). Int J Methods Psychiatr Res 27:e1596. https://doi.org/10.1002/mpr.1596

23. Critchley HD, Wiens S, Rotshtein P, et al (2004) Neural systems supporting interoceptive awareness. Nat Neurosci 7:189-95

24. Starmans C, Bloom P (2012) Windows to the soul: Children and adults see the eyes as the location of the self. Cognition 123:313-318. https://doi.org/10.1016/j.cognition.2012.02.002

25. Limanowski J, Hecht H (2011) Where do we stand on locating the self? Psychology 02:312-317. https://doi.org/10.4236/psych.2011.24049

26. Fetterman AK, Robinson MD (2013) Do you use your head or follow your heart? Self-location predicts personality, emotion, decision making, and performance. J Pers Soc Psychol 105:316334. https://doi.org/10.1037/a0033374

27. Porciello G, Daum MM, Menghini C, et al (2016) Not That Heart-Stopping After All: VisuoCardiac Synchrony Does Not Boost Self-Face Attribution. PLoS ONE 11:e0160498. https://doi.org/10.1371/journal.pone.0160498

28. Deroualle D, Toupet M, van Nechel C, et al (2017) Anchoring the Self to the Body in Bilateral Vestibular Failure. PLoS ONE 12:e0170488. https://doi.org/10.1371/journal.pone.0170488

29. Strupp M, Arbusow V, Dieterich M, et al (1998) Perceptual and oculomotor effects of neck muscle vibration in vestibular neuritis. Ipsilateral somatosensory substitution of vestibular function. Brain 121:677-85.

30. Zigmond AS, Snaith RP (1983) The hospital anxiety and depression scale. Acta Psychiatr Scand $67: 361-370$

31. Limanowski J, Hecht H (2011) Where Do We Stand on Locating the Self? Psychology 02:312. https://doi.org/10.4236/psych.2011.24049

32. Peirce JW (2007) PsychoPy - Psychophysics software in Python. J Neurosci Methods 162:8-13 
33. Aron A, Aron EN, Smollan D (1992) Inclusion of Other in the Self Scale and the structure of interpersonal closeness. Journal of Personality and Social Psychology 63:596-612. https://doi.org/10.1037/0022-3514.63.4.596

34. Ring C, Brener J (2018) Heartbeat counting is unrelated to heartbeat detection: A comparison of methods to quantify interoception. Psychophysiology 55:e13084.

https://doi.org/10.1111/psyp.13084

35. Garfinkel SN, Seth AK, Barrett AB, et al (2015) Knowing your own heart: distinguishing interoceptive accuracy from interoceptive awareness. Biol Psychol 104:65-74. https://doi.org/10.1016/j.biopsycho.2014.11.004

36. Jauregui-Renaud K, Yarrow K, Oliver R, et al (2000) Effects of caloric stimulation on respiratory frequency and heart rate and blood pressure variability. Brain Res Bull 53:17-23. https://doi.org/10.1016/s0361-9230(00)00304-x

37. Garfinkel SN, Manassei MF, Hamilton-Fletcher G, et al (2016) Interoceptive dimensions across cardiac and respiratory axes. Philos Trans R Soc Lond, B, Biol Sci 371:. https://doi.org/10.1098/rstb.2016.0014

38. Trousselard M, Barraud P, Nougier V, et al (2004) Contribution of tactile and interoceptive cues to the perception of the direction of gravity. Brain Res Cogn Brain Res 20:355-362. https://doi.org/10.1016/j.cogbrainres.2004.03.008

39. Lopez C, Nakul E, Preuss N, et al (2018) Distorted own-body representations in patients with dizziness and during caloric vestibular stimulation. J Neurol 265:86-94. https://doi.org/10.1007/s00415-018-8906-8

40. Lopez C, Elzière M (2018) Out-of-body experience in vestibular disorders - A prospective study of 210 patients with dizziness. Cortex 104:193-206. https://doi.org/10.1016/j.cortex.2017.05.026

41. Ferrè ER, Lopez C, Haggard P (2014) Anchoring the self to the body: vestibular contribution to the sense of self. Psychol Sci 25:2106-2108. https://doi.org/10.1177/0956797614547917

42. Deroualle D, Borel L, Devèze A, Lopez C (2015) Changing perspective: The role of vestibular signals. Neuropsychologia 79, Part B:175-185. https://doi.org/10.1016/j.neuropsychologia.2015.08.022

43. Candidi M, Micarelli A, Viziano A, et al (2013) Impaired mental rotation in benign paroxysmal positional vertigo and acute vestibular neuritis. Front Hum Neurosci 7:783. https://doi.org/10.3389/fnhum.2013.00783

44. Claparède E (1925) Note sur la localisation du moi. Archives de Psychologie 19:172-182

45. Tsakiris M, Tajadura-Jiménez A, Costantini M (2011) Just a heartbeat away from one's body: interoceptive sensitivity predicts malleability of body-representations. Proc Biol Sci 278:24702476. https://doi.org/10.1098/rspb.2010.2547

46. Tschan R, Wiltink J, Adler J, et al (2013) Depersonalization experiences are strongly associated with dizziness and vertigo symptoms leading to increased health care consumption in the German 
general population. J Nerv Ment Dis 201:629-635.

47. Kolev OI, Georgieva-Zhostova SO, Berthoz A (2014) Anxiety changes depersonalization and derealization symptoms in vestibular patients. Behav Neurol 2014:847054. https://doi.org/10.1155/2014/847054

48. Noel J-P, Pfeiffer C, Blanke O, Serino A (2015) Peripersonal space as the space of the bodily self. Cognition 144:49-57. https://doi.org/10.1016/j.cognition.2015.07.012

49. Nakul E, Orlando-Dessaints N, Lenggenhager B, Lopez C (2020) Measuring perceived selflocation in virtual reality. Sci Rep 10:6802. https://doi.org/10.1038/s41598-020-63643-y 
A
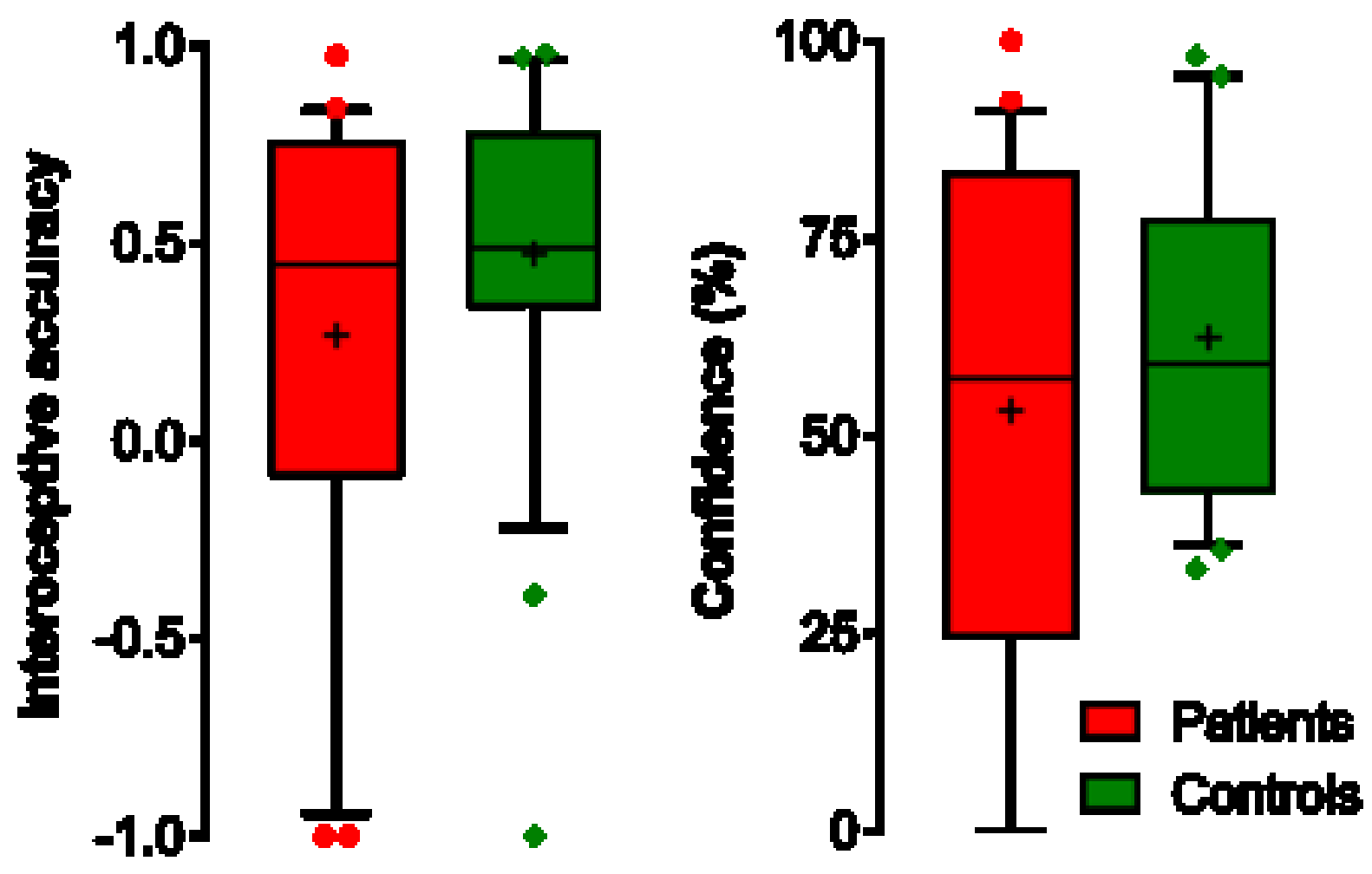

B

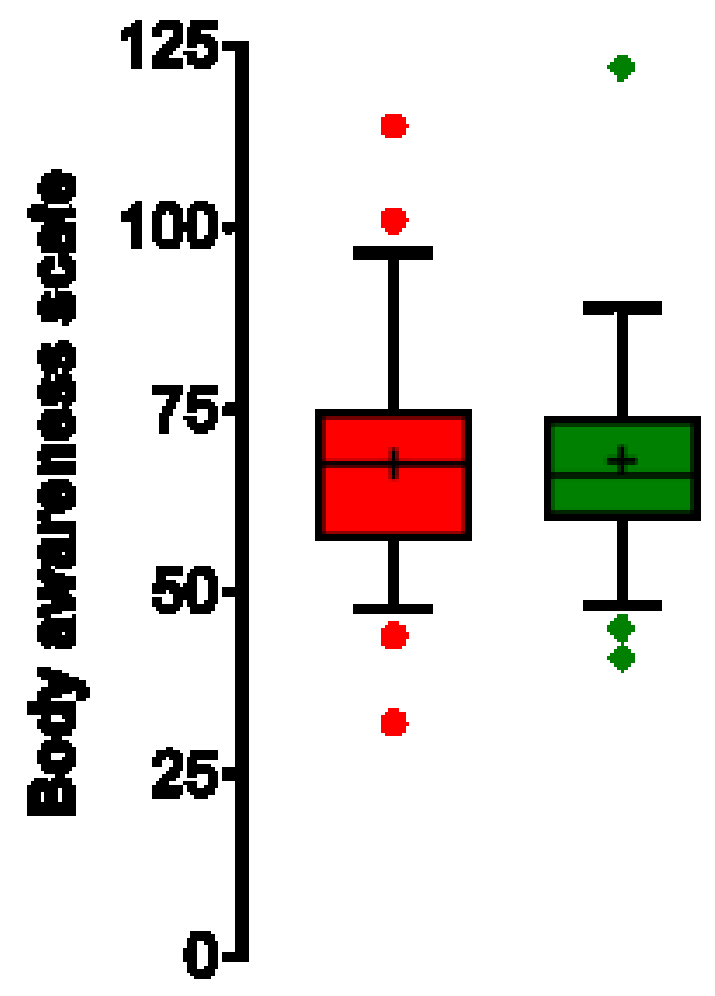



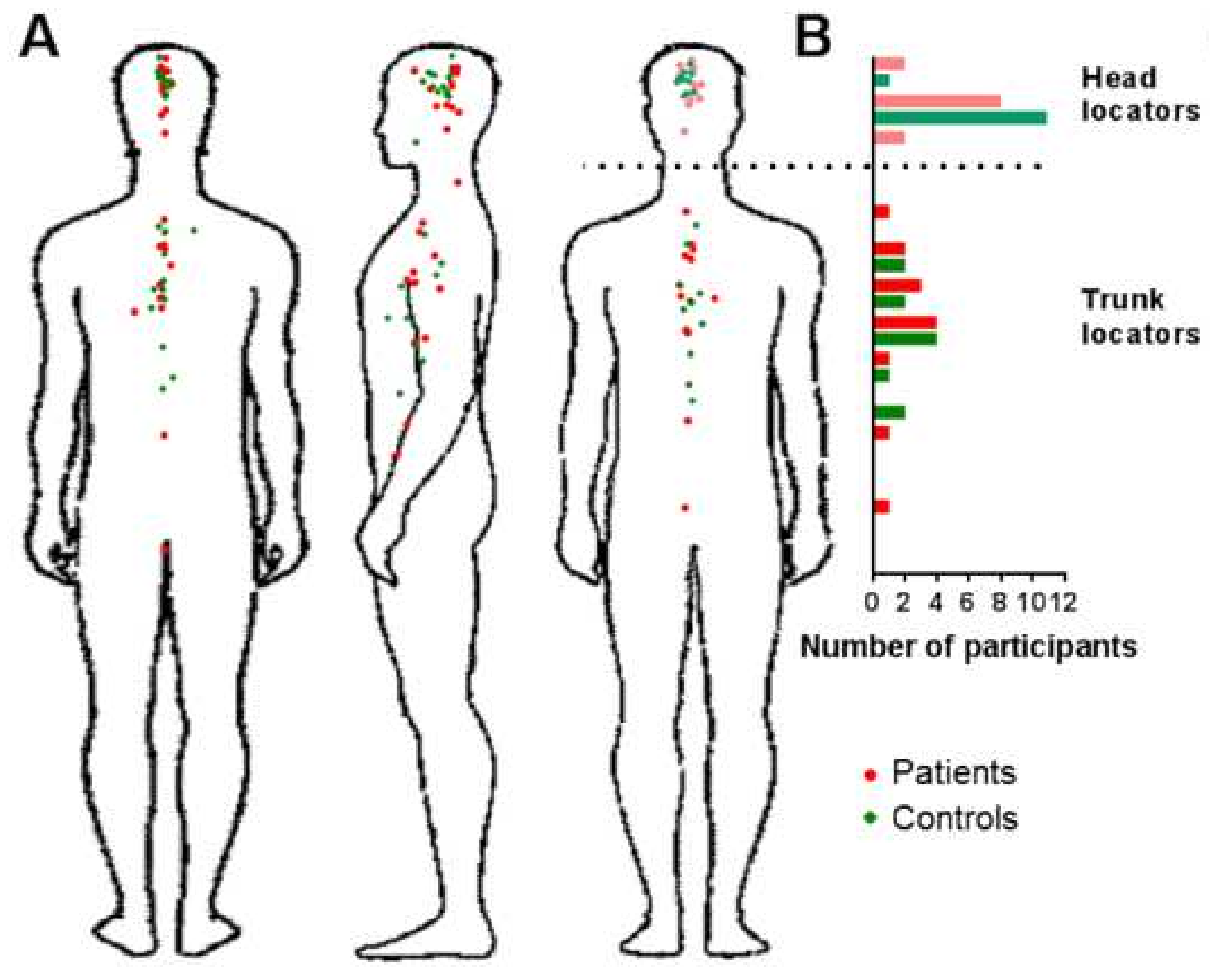

C
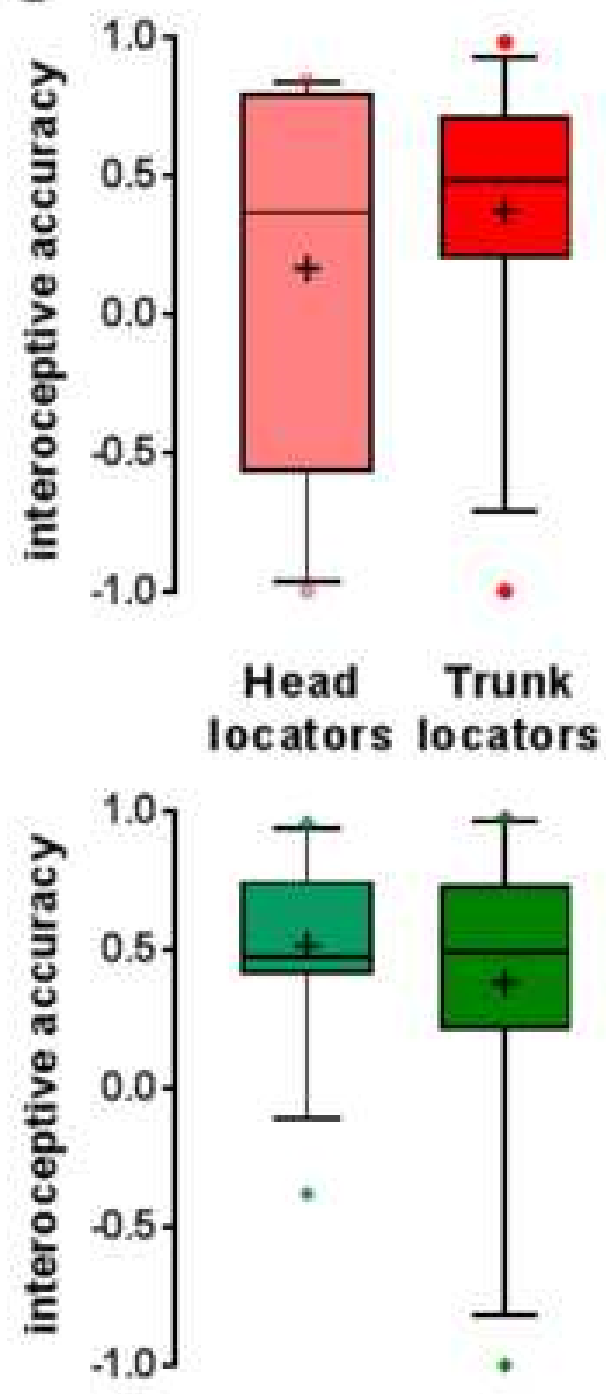

- Patients

- Controls

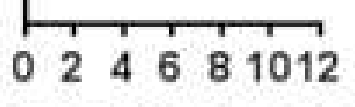

umber of participants
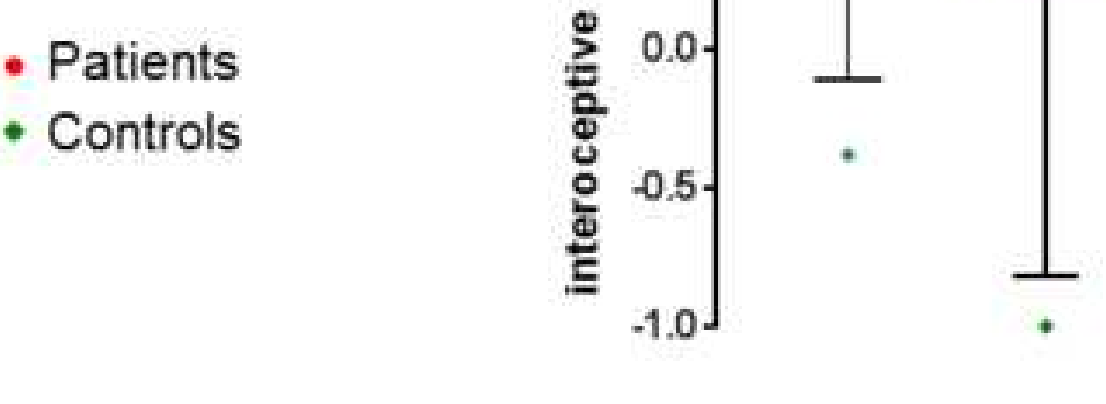


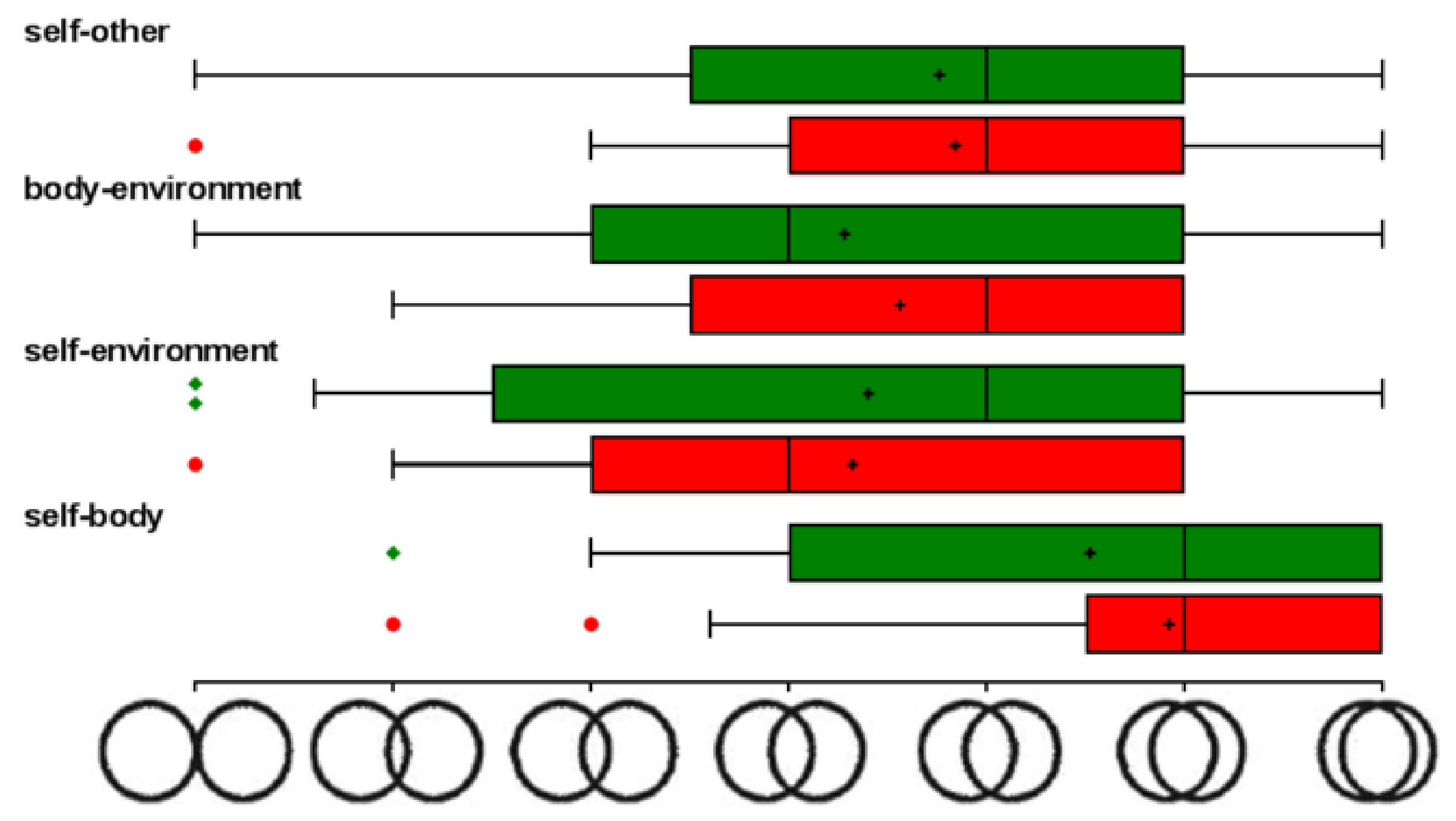


A
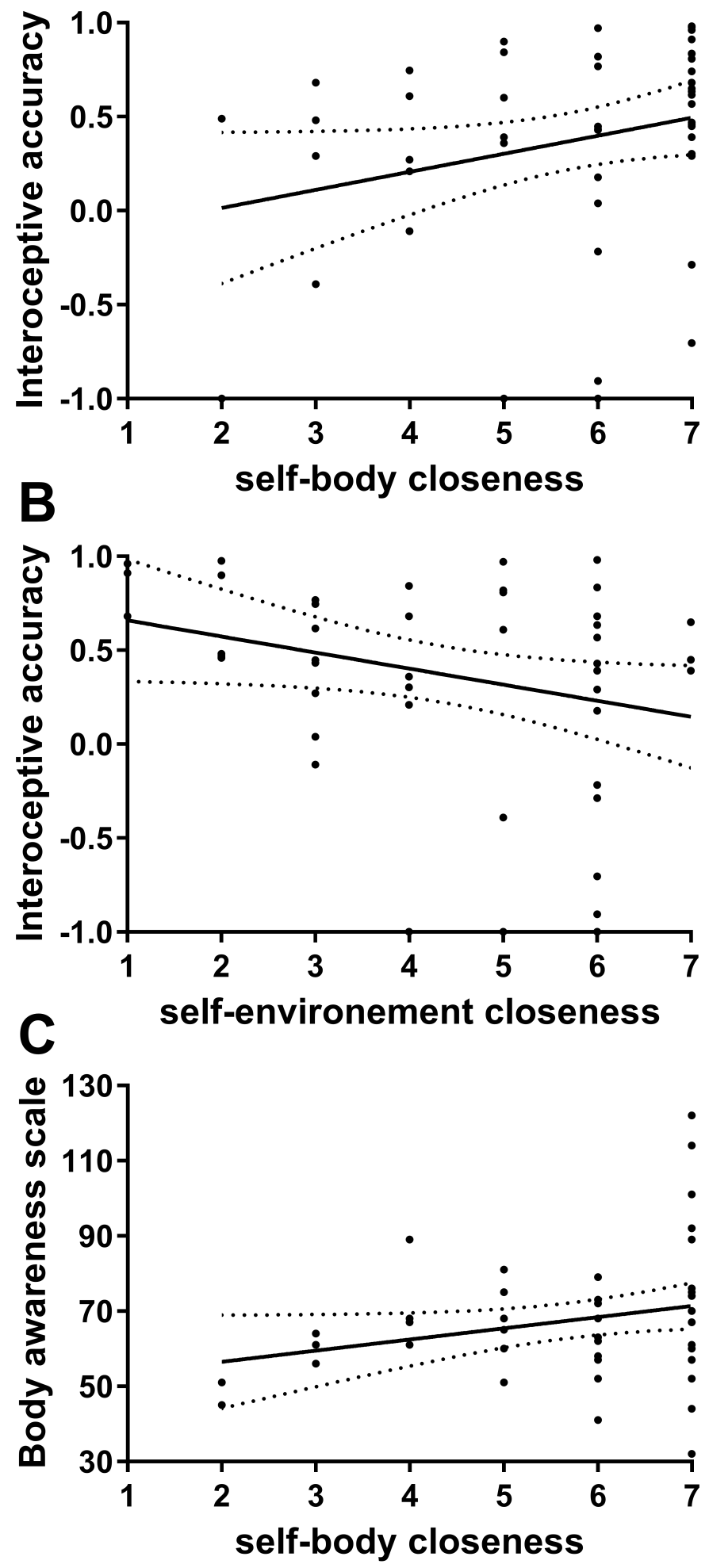


\begin{tabular}{lccc}
\hline & Patient & Controls & Statistics \\
\hline Males/Females (n) & $11 / 14$ & $11 / 14$ & - \\
\hline Age (years) & $60 \pm 13$ & $61 \pm 11$ & $\mathrm{t}=-0.43, \mathrm{p}=0.67$ \\
\hline Education (\%) & & & \\
Level 1 & 8 & 4 & \\
Level 2 & 4 & 8 & $\chi=3.72, \mathrm{p}=0.45$ \\
Level 3 & 16 & 20 & \\
Level 4 & 32 & 12 & \\
Level 5 & 40 & $24 \pm 3$ & $\mathrm{t}=-0.10, \mathrm{p}=0.92$ \\
\hline Body-mass index & $24 \pm 4$ & $7.1 \pm 3.1$ & $\mathrm{t}=-0.63, \mathrm{p}=0.53$ \\
\hline Anxiety score & $8.3 \pm 2.5$ & $3.7 \pm 2.6$ & $\mathrm{t}=0.34, \mathrm{p}=0.74$ \\
\hline Depression score & $4.0 \pm 2.4$ & & \\
\hline
\end{tabular}

Table 1. Demographic and clinical characteristics of BVP patients and healthy controls. Highest education level according to the French education system; Level 1: before high school; Level 2: accomplished high school; Level 3: two years after high school; Level 4: Bachelor's degree, Level 5: Master's degree, Engineering degree, $\mathrm{PhD}, \mathrm{MD}$. Mean $\pm \mathrm{SD}$. Anxiety and depression scores were measured with the Hospital Anxiety and Depression scale. 